\title{
Review
}

\section{Glyoxalases in Urological Malignancies}

\author{
Cinzia Antognelli ${ }^{*}$ and Vincenzo Nicola Talesa ${ }^{1}$ \\ 1 aDepartment of Experimental Medicine, University of Perugia ; cinzia.antognelli@unipg.it; \\ vincenzo.talesa@unipg.it \\ * Correspondence: cinzia.antognelli@unipg.it; Tel.: +39-075-585-8354
}

\begin{abstract}
Urological cancers include a spectrum of malignancies affecting organs of the reproductive and/or urinary systems, such as prostate, kidney, bladder, and testis. Despite improved primary prevention, detection and treatment, urological cancers are still characterized by an increasing incidence and mortality worldwide and although advances have been made toward understanding the molecular basis of these diseases, a complete insight of the pathogenic mechanisms is still a research challenge for defining safer pharmacological therapies and prognostic factors, especially for the metastatic stage of these neoplasms for which no effective therapies exist. Glyoxalases are enzymes that catalyzes the glutathione-dependent metabolism of cytotoxic methylglyoxal (MG), thus protecting against cellular damage and apoptosis. They are generally overexpressed in numerous cancers as a survival strategy by providing a safeguard through enhancement of MG detoxification. Increasing evidence suggest that glyoxalases, especially Glo1, would act as oncogenes in urological malignancies and be central to their initiation, growth and progression. In this review, we highlight the critical role of glyoxalases as regulators of tumorigenesis in the prostate through modulation of various critical signaling pathways, and provide an overview of the current knowledge on glyoxalases in bladder, kidney and testis cancers. We also discuss the promise and challenges for Glo1 inhibitors as future anti-PCa therapeutics and the potential of glyoxalases as biomarkers for PCa diagnosis.
\end{abstract}

Keywords: Glyoxalases; urological malignancies; prostate cancer; kidney cancer; bladder cancer testicular cancer.

\section{Introduction}

Urological cancers include a spectrum of malignancies affecting organs of the reproductive and/or urinary systems, such as prostate, kidney, bladder, and testis. Excluding testicular cancer, they are among the ten most frequent malignancies in man [1]. According to the most recent cancer statistics, renal, bladder and prostate cancers constitute more than 33\% of all cancers in the United States in 2016 [2]. Despite improved primary prevention, early detection and treatment, urological cancers are still characterized by an increasing incidence and mortality worldwide [1] and although advances have been made toward understanding the molecular basis of these diseases, a complete insight of the pathogenic mechanisms is still a research challenge for defining safer pharmacological therapies and prognostic factors, especially for the metastatic stage of these neoplasms for which no effective therapies exist. Prostate cancer (PCa) is a major health concern in the older male populations all over the world and is the sixth more common cause of cancer related deaths in the world [3]. Androgen receptor plays a crucial role in the development of $\mathrm{PCa}$ and androgen deprivation therapy is the first line therapy for newly diagnosed PCa patients [4]. Nevertheless, most patients progress to castrationresistant $\mathrm{PCa}$, very often associated with metastases [5]. Bladder cancer (BCa) incidence is three times higher among males than females [6]. Nearly 386000 newly diagnosed cases and about 150000 deaths are reported annually worldwide [4]. Renal cell carcinoma (RCC) is the eighth most common cancer in the United States [7] and its incidence is steadily rising in most areas of the world [8]. Total or partial nephrectomy is the optimal primary treatment. Nevertheless, RCC recurs in $20-40 \%$ of patients after resection, which is associated with a worse tumor stage and grade [9]. To further exacerbate the situation, at least some malignancies of the urinary tract are age-related, therefore, it is very plausible 
that their incidence may continue to rise as a result of global population aging. Multiple factors are involved in the genesis and progression of urological malignancies, including hypoxia, hormone influence, genetic predisposition, oxidative and glycative stresses and inflammation [10-18]. With regard to oxidative stress, glycative stress and inflammation a complex interplay is known to exist between these biological phenomena, so that each of them fuels the other in a fine-tuned regulatory circuitry. A crucial common nodal factor in this interplay is represented by methylglyoxal (MG), an endogenous dicarbonyl metabolite [19], formed during cell metabolisms, especially glycolysis, so that MG is particularly abundant in actively proliferating cancerous cells, that primarily rely on glucose metabolism to support their elevated growth demands, according to the so-called Warburg effect" [20]. MG is a potent glycating agent [19], able to rapidly and spontaneously react with amino group in proteins and with DNA, irreversibly generating a family of heterogeneous compounds called advanced glycation end products (AGEs) [21]. Directly or through AGEs, MG is also able to generate reactive oxygen species (ROS) [22] and/or induce inflammation [23], all these events ultimately leading to cell death [24-26] or transformation [27]. Intracellular concentrations of MG are strictly regulated by Glyoxalases [29], cellular enzymes whose increased expression and activity in tumor tissues and/or cell lines [29-32], including those from the urogenital tract [33-37], have been largely documented [29-37], suggesting a pivotal role for them in human tumorigenesis. Glyoxalases upregulation in tumors would be a defense strategy adopted by cancerous cells towards increased intracellular cytotoxic MG. In particular, Glo1 increased expression is likely due to an increased requirement to protect the tumor proteome against a local, relatively high flux of pro-apoptotic and anti-proliferative MG formation [28]. Here, we review the role of Glyoxalases in the onset and progression of human urogenital malignancies and Glyoxalases potential as targets for anticancer drug development and biomarkers for diagnosis or prognosis of urological cancers.

\section{Glyoxalases}

Glyoxalases comprise two enzymes, named Glyoxalase 1 (Glo1) and Glyoxalase 2 (Glo2). The two enzymes have been historically described to work in tandem as part of a system where Glo1 catalyzes the isomerization of the hemithioacetal formed non-enzymatically by the reaction of MG with reduced glutathione (GSH) into S-D-lactoylglutathione, and Glo2 the hydrolysis of S-Dlactoylglutathione to D-lactate, thereby reforming GSH [21] (Figure 1). 


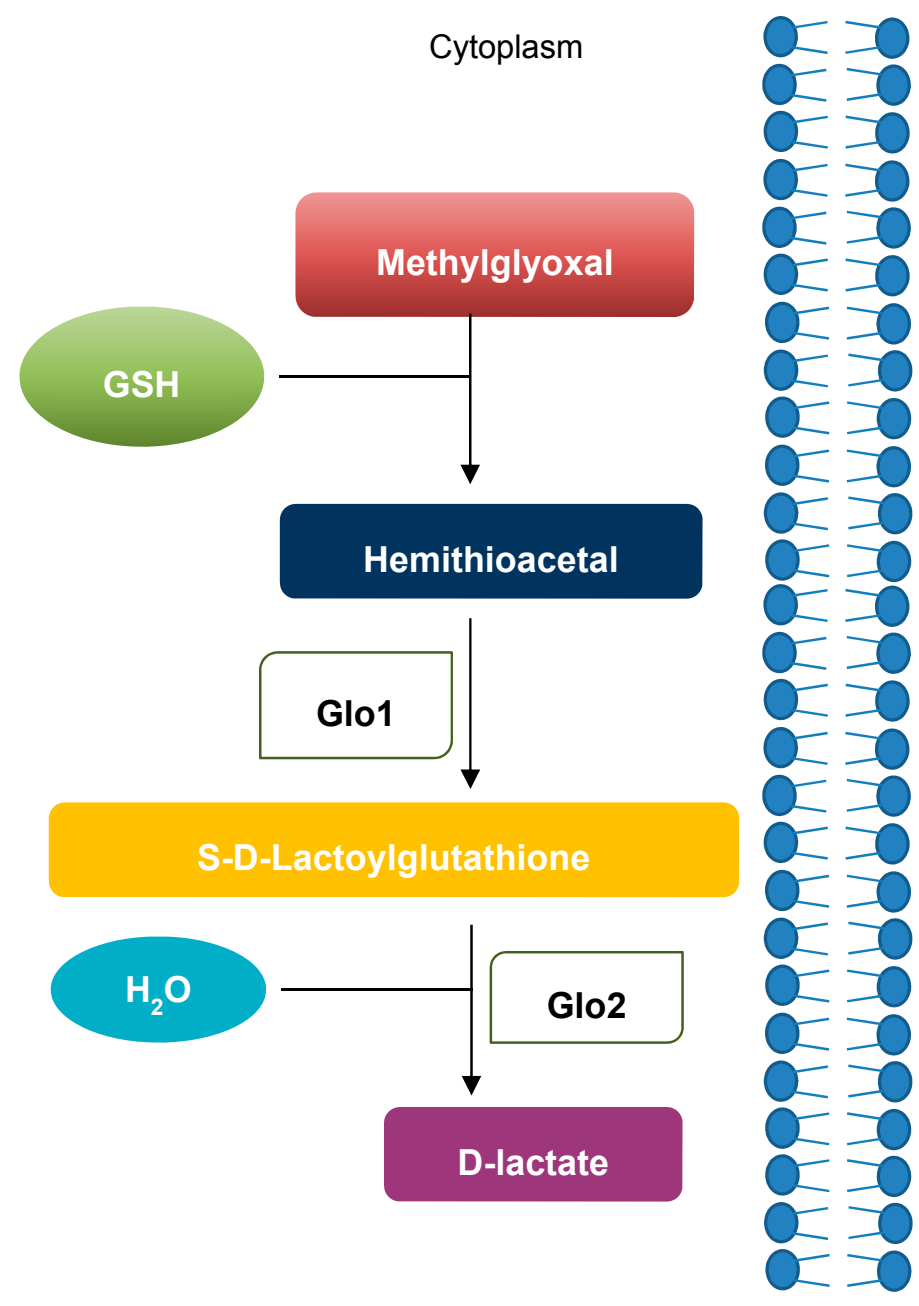

Figure 1. Glyoxalases.

Glo1 activity can be regulated through several mechanisms including gene expression regulation $[21,26,35,36]$ and post-translational modifications such as phosphorylation, NO-mediated modification and glutathionylation [21,38-41]. Glo2 expression can be upregulated by the transcription factors p63 and p73 [42], steroid hormones [35], androgen receptor [37] and PTEN/PI3K/AKT/mTOR signalings [36]. Extensive information about physical and chemical properties of glyoxalases has been largely described in excellent previous reviews [43-48].

\section{Prostate cancer (PCa)}

\subsection{Glo1}

The first interest on the role of Glo1 in PCa dates back to the 1990's, when Ayoub et al. [49] and Di Ilio et al. [50] measured Glo1 specific activity in human tumor cell lines in vitro or cancerous and noncancerous tissues. Although PCa tissues were poorly represented and PCa cell lines not clearly described, those results seemed to suggest a role for Glo1 in tumorigenesis, being its enzymatic activity higher in cancerous cells compared with non-malignant ones. The first seminal observations of the potential role of Glo1 in PCa onset was done later by Davidson et al. [51] who performed a quantitative PAGE-based Glo1 assay on 22 PCa and 10 normal prostate tissues to assess its activity, in the attempt to elicit its possible clinical implication in this neoplasm. These results demonstrated 
that Glo1 activity differed substantially and significantly between non-cancerous and cancerous specimens, with a densitometric analysis showing differences in the range from 5- to 8-fold in PCa compared with non-cancerous samples. Notably, a distinctly high activity of Glo1 was observed in 22 out of 22 PCa specimens and none to little activity in 10 out of 10 non-malignant specimens. Although preliminary, these results opened new pathways of investigation towards the study of Glo1 expression, either at mRNA, protein or functional level, in PCa tissues and normal counterparts or in differently aggressive and invasive PCas [36,37,52-55]. Overall, these studies strongly indicated Glo1 as a novel important protein involved in PCa genesis and progression, thus suggesting an oncogenic role for this enzyme in this neoplasm. Overexpression of Glo1 in PCa cells would aim at maintaining low intracellular levels of the glycolysis-derived cytotoxic metabolite MG, as a survival defense strategy. Indeed, in vitro studies performed with PCa cell lines, causatively demonstrated this role $[24,25,36]$, in addition to pointing out a pro-survival function for this protein by eluding apoptosis in a mechanism involving the desensitization of NF-kB pathway [25]. Only one study, based on the proteomic analysis of normal and malignant prostate tissues, identified Glo1 among the proteins lost in PCa [56]. The traditionally accepted oncogenic role of Glo1 in PCa has reasonably made scientists think that it could be considered, on the one hand, as a marker for diagnosis of PCa and, on the other hand, a potential target for the development of alternative anti-cancer or chemotherapeutic agents for PCa, which were indeed confirmed by some studies. In particular, Chavan et al. [57] suggested serum Glo1 as a supplemental biomarker to differentiate between malignant and non-malignant diseases of the prostate in patients with PSA in the range of 4-20 ng/ml. Similarly, we first explored the association of Glo1 A111E functional polymorphism, associated with a loss-of-function mutation and for which we demonstrated evidence of a biological plausibility, providing an additional candidate for risk assessment in PCa patients and an independent prognostic factor for survival [58]. Although further investigations with a larger number of participants are absolutely required to confirm these findings, these explorative studies pioneered researches important for PCa diagnosis and encourage further progress in this field. So far, the gold standard diagnosis for urological neoplasms is pathological diagnosis and the early screening methods are rare. Some existing biomarkers such as prostate-specific antigen (PSA) may be useful in PCa screening, however, due to its poor specificity, this is associated with over-diagnosis and over-treatment, which limits its application [59-61]. With regard to Glo1 as drug target, Sharkey et al. [62] provided the first demonstration that a competitive inhibitor of Glo1 effectively inhibited the growth of PCa tumors in mice, when delivered as the diethyl ester prodrug. Even, the antitumor effects of this Glo1 inhibitor were comparable to those observed with the standard antitumor agents. Carmustine (BCNU) is an anticancer agent and another putative inhibitor of Glo1, while B-glucan is a unique, nontoxic polysaccharide extracted from mushrooms. In 2002 it was demonstrated a sensitized cytotoxic effect of BCNU with $B$-glucan in PC3 PCa cells, which was associated with a drastic (approximately $80 \%$ ) inactivation of Glo1 [63]. More recently, it has been also reported that curcumin inhibits Glo1 with the consequent intracellular accumulation of non-tolerable levels of MG and GSH, that, by modulating various metabolic cellular pathways, hamper malignant cells growth [64]. Finally, more recently, Baunacke et al. [53] identified ethyl pyruvate as agent targeting Glo1 and defanging some malignancy-associated properties of PCa cells and Valenti et al. [65] found that 3-bromopyruvate induced rapid human PCa cell death by affecting, among the others, Glo1.

\subsection{Glo2}

Differently from Glo1, the role of Glo2 in PCa has been scarcely investigated [36,37,49,50]. Ayoub et al. [49] and Di Ilio et al.[50] first attempted to evaluate Glo2 enzymatic activity in human PCa cell lines and tissues. They found that Glo2 enzyme activity in cancerous cells was not so different from that of non-cancerous cells. However, the scanty number of PCa samples considered in those studies significantly limited a reliable interpretation on the biological significance of Glo2 in this neoplasia. Only recently we causatively demonstrated a role of Glo2 in this neoplasia [36,37] providing in vivo and, in vitro, evidence for a role of this enzyme in prostate carcinogenesis. In particular, we showed that Glo2 was selectively expressed in PCa but not in the luminal compartment of the adjacent benign 
epithelium consistently in all the examined cases $(n=20)$. Moreover, we demonstrated that Glo2 expression in malignant prostate cells was dependent on androgen receptor, in line with another previous exploratory study by our group [35], and was aimed at stimulating cell proliferation and eluding apoptosis through a mechanism involving the p53-p21 axis. Hence, our results demonstrated for the first time a role of $\mathrm{Glo} 2$ in prostate tumorigenesis and the associated mechanism. Both genetic and environmental factors have been demonstrated to be involved in PCa pathogenesis [66]. Nevertheless, the molecular biology and mechanisms of prostate carcinogenesis remain to be further elucidated in order to identify additional diagnostic factors. As above mentioned, while the possibility of biomarkers for PCa has been investigated for some molecules, their prospects in clinical application still need to be further evaluated $[60,61,67]$. The need for additional biomarkers that supplement PSA is urgently needed. Since we demonstrated that the luminal Glo2 is able to drive prostate tumorigenesis and that it is not expressed in these luminal benign cells but only in PCa, we proposed that it might represent a novel marker in the pathologic diagnosis of early PCa, by distinguishing between benign and malignant lesions. In fact, the possible observation of Glo2 staining in the luminal cells of the benign gland during the pathologic diagnosis might be indicative of an initial/ongoing neoplastic transformation process. In addition, we found in tissue sections that Glo2 was also intensely expressed in the basal cells of benign glands, even though this basal cells localized Glo2 was not involved in PCa genesis. The histological diagnosis of PCa, at least in difficult cases, is based upon prostate gland architectural and cytological features, with basal cell loss as a hallmark of malignancy. When the growth pattern is obscured - as can be the case in core needle biopsies with few suspicious glands - assessing these basal cells immunohistochemically is the usual auxiliary approach in confirming or excluding malignancy [68]. A variety of basal cell markers have been suggested. The standard markers used routinely in surgical pathology are high molecular weight cytokeratins (e.g. 34ßE12, CK5/6) or p63, both of which stain basal cells with high sensitivity and specificity [68]. However, in some cases false-negative staining may occur because of patchy cytoplasmic staining, making a definitive diagnosis difficult. We suggested that Glo2 immunostaining could represent a novel additional marker for basal cells in the benign prostate and its absence in malignant cells, consequent to the loss of basal cells, might have been useful as an adjuvant method to facilitate the pathological diagnosis of PCa. In particular, since the loss of basal cells is an early event during tumorigenesis, we believe that Glo2 might be especially important for the early diagnosis of PCa. Glo2, together with Glo1, belongs to the glyoxalase pathway. However, a demonstration of a causative relationship between the two has never been demonstrated. In our study, the results from IHC showed an opposite localization of Glo1 with respect to Glo2. In all 20 cases of non-tumoral glands Glo1 staining consistently was observed only in the luminal but not in the basal cells, suggesting an independent role of the two proteins. Moreover, when attempting to demonstrate a possible role of Glo1 in association with Glo2 in PCa genesis via cell growth control we did not find any robust dependency. We suggest that Glo2 involvement in prostate tumorigenesis is independent of Glo1, at least as far as cell growth control is concerned, and/or that Glo1 may be involved in PCa genesis via the control of other tumorigenesis-related biological processes, possibly induced by factors in the cancer microenvironment, which is under investigation in our laboratory. We recently found that Glo2, this time in cooperation with Glo1, is also involved in human PCa progression as part of a mechanism driven by PTEN/PI3K/AKT/mTOR signaling with involvement of PKM2 and estrogen receptor alpha [36]. In particular, we found that both enzymes were upregulated in both aggressive PCa tissues and cell lines and this up-regulation was aimed at maintaining low intracellular levels of the glycolysis-derived cytotoxic metabolites MG and LSG, very likely as a survival defense strategy. Importantly, Glo2 silencing did not alter the behavior of benign cells, suggesting that targeting this protein might represent a strategy to selectively inhibit advanced PCa. 
6 of 12

\section{Renal cancer (RCa)}

\subsection{Glo1}

Back in 1995, Di Ilio and colleagues evaluated Glo1 enzyme activity in the cytosolic fractions prepared from 15 samples of neoplastic and healthy kidney tissues from patients with primary renal cell carcinoma [50]. However, no significant differences were observed. Ten years later, we investigated Glo1 expression, at both mRNA and functional level, in a human renal carcinoma (clear cell adenocarcinoma, CCA), known to be strongly resistant to chemotherapeutic drugs, and in pairmatched normal tissues [34]. This study clearly provided evidence for significantly higher (about nine-fold) levels of Glo1 mRNA and activity (2.5-fold) and markedly reduced intracellular amount of pro-apoptotic MG in CCA than in control tissues, suggesting a mechanism explaining, at least in part, the refractoriness of CCA to a number of apoptosis-inducing chemotherapy treatments. In fact, despite the discovery of a number of new cytotoxic agents in the recent years, kidney cancer treatment is still unsatisfactory, mainly due to a marked resistance of this tumor towards these drugs. Hence, we suggested that the synthesis of new molecules selectively down-regulating Glo1 expression in cancer cells might have been an alternative method of therapeutic treatment for this neoplasia, in particular CCA. Our study was later extended by Tanaka and colleagues who studied Glo1 expression in human renal tumor cell lines with diverse metastatic potential [69]. By using twodimensional electrophoresis followed by liquid chromatography-tandem mass spectrometry, they found that protein Glo1 appeared to be directly proportional to the metastatic potential of the studied clones, in addition to be the only protein which consistently varied with the metastatic potentials of CCR clones: Glo1 was increased in highly metastatic cell lines compared with those with lower metastatic potential and this was confirmed by western blot analysis. These results, in agreement with ours [34], suggested that the increased Glo1 expression in more aggressive CCR cells would have provided advantages to the metastatic potential in aggressive clones, and that Glo1 inhibition might have been a therapeutic potential for the treatment of metastatic CCR. A novel mechanism underlying increased Glo1 expression in tumors is GLO1 gene amplification [70]. A quite recent study demonstrated a direct link between GLO1 gene amplification, overexpression and increased sensitivity to Glo1 inhibition in $6.5 \%$ of RCa, and suggested that cell lines with GLO1 amplification were significantly more sensitive to GLO1 inhibition than those without. Moreover, it was suggested that Glo1 might represent a useful target for therapy in cancers with Glo1 amplification, as RCa. As to studies on the association of Glo1 A111E polymorphism with the risk of RCa, they have been limited to only one study in a cohort of 214 patients with CCA [71]. This study demonstrated the link of E111A (419A/C) Glo1 SNP to the presence of CCR. In particular, a higher frequency of the C allele and genotype CC in patients with CCR was observed in comparison with the control group. This result is in accordance with the fact that the $\mathrm{C}$ allele is linked to a higher activity of GLO1, which has been previously observed [34]. However, although this study clearly demonstrated Glo1 involvement in kidney cancer, it presented some potential limitations, the most important of which was the lack of a biological plausibility, since Glo1 activity was not measured either in tissue or blood samples.

\subsection{Glo2}

A clear connection between Glo2 and RCa was first established in 1995 when once again Di Ilio and colleagues [50] evaluated Glo2 enzyme activity in the cytosolic fractions from 15 samples of neoplastic or healthy kidney tissues of patients with primary RCC. In this study Di Ilio et al. revealed a significant about 4-fold lower Glo2 activity in malignant than in non-malignant kidney tissues. Only one study by our group followed this exploratory observation [34]. In agreement with Di Ilio et al. we demonstrated a significant decrease of Glo2 enzyme activity nearly halved in the tumor compared with non-tumor tissues. 


\section{Bladder cancer (BCa)}

\subsection{Glo1 and Glo2}

Scanty information is available on Glyoxalases in BCa [33,50]. A study [50] reported Glyoxalases activity in 6 samples of normal bladder and in the tumor and non-tumor samples of 8 bladders. Large inter-individual variations were observed in both Glo1 and Glo2 activities which, according to the authors, made the definition of normal baseline level difficult to set. Although no significant difference in Glo1 mean activity was found between tumor and non-tumor samples of BCa, relatively higher tumor Glo1 activity, compared with the corresponding non-tumor tissues, was found in five samples. As to Glo2, no significant differences were observed between the cancerous component and the normal counterpart. Next, we studied Glo1 and Glo2 gene expression and ezyme activity in human BCa compared with the corresponding normal mucosa [33]. In particular, samples of these tissues were collected from 26 patients with superficial (SBCa) or invasive bladder cancer (IBCa). Both Glo1 mRNA expression and activity were significantly increased in SBCa samples, while they remained unchanged in IBCa samples, compared with the normal mucosa. Conversely, Glo2 showed a higher activity in the tumor (either SBCa or IBCa samples) versus normal tissues. These results suggested that the differing levels of Glo1 activity level and gene expression between the SBCa and IBCa samples might have helped in their differential diagnosis.

\section{Testis cancer (TCa)}

\subsection{Glo1 and Glo2}

As far as we know, only Di Ilio and colleagues reported on the activity of Glyoxalases in TCa more than twenty years ago [50]. However, with the exception of one sample, they found Glo1 and Glo2 activity essentially identical between tumor and non-tumor samples.

\section{Concluding remarks and future directions}

Studies so far performed on the role of glyoxalases in urological cancers mainly focused on PCa. These studies pointed out the oncogenic role of Glo1, especially during PCa progression, according to which increased expression and activity of Glo1 would be permissive for survival and growth of tumors with relatively high glycolytic rates and related high fluxes of MG formation. Hence, in PCa, utilization of specific inhibitors of Glo1 might indeed serve as an effective anticancer strategy. Several natural compounds have been proposed as Glo1 inhibitors attenuating the growth of PCa [53, 62-65]. In addition, these studies demonstrated that also Glo2, together with Glo1, represents a novel mechanism in PCa progression [36] and, more importantly, that Glo1/Glo2 silencing did not alter the behavior of benign cells [36], thus suggesting that targeting glyoxalases metabolic pathway may represent a strategy to selectively inhibit advanced PCa. Future in vivo studies needs to be urgently performed to validate these important conclusions, in order to translate them to a potential clinical application. If the traditional cooperative role of Glo1/Glo2 has been clearly pointed out in PCa progression, in PCa genesis it appears that Glo1 and Glo2 have independent roles, not working sequentially as a metabolic system, thus contributing on raising again the question whether the glyoxalase pathway is really a pathway [44] or, at least, whether it is that only in certain conditions. Additional researches are needed to shed new light into the role of these intricate and intriguing proteins in PCa and, more in general, in the oncologic ambit. Finally, studies performed on the role of Glyoxalases in PCa demonstrated that Glo1 and Glo2 may represent novel adjuvant marker in the pathological diagnosis of advanced and early $\mathrm{PCa}$, respectively. We hope that urologist and oncologist, thanks to these new advances, may find renewed interest on these proteins, frequently overlooked in cancer research. By far less studied is the role of glyoxalases in renal and bladder cancers. The scarce information available, although to be further corroborated, would suggest the potential employment of these proteins as biomarkers of these neoplastic diseases, whose incidence 
continues to rapidly increase worldwide and for the early detection and follow up of which no biomarkers are currently available [14]. RCa and BCa lack specific predictive biomarkers and only some symptoms might have some effects in finding the existence of cancer [72,73]. The study of the role of glyoxalases in TCa is today pratically still a "virgin field". TCas are a group of heterogeneous, biologically diverse and clinically challenging neoplasms. Despite the relatively low incidence and mortality rates, a subgroup of patients with disseminated disease relapse after conventional therapy and have a dismal prognosis. Moreover, TCs afflict mostly young men and have therapeutic peculiarities, with some patients showing resistance to cisplatin-based treatments and others being troubled by irreversible side effects, such as infertility [74,75]. Due to these valid reasons, researchers are strongly encouraged to initiate studies also in this ambit.

Research on Glyoxalases has experienced frequent and surprising turns since they were discovered. Research on Glyoxalases in urological malignancies might unravel, as already done in the past, novel and interesting roles of these proteins and bring them to the limelight once more.

Conflicts of Interest: The authors declare no conflict of interest

$\begin{array}{ll}\text { Abbreviations } & \\ \text { Glo1 } & \text { Glyoxalase } 1 \\ \text { Glo2 } & \text { Glyoxalase } 2 \\ \text { MG } & \text { Methylglyoxal } \\ \text { AGEs } & \text { Advanced glycation end products } \\ \text { PCa } & \text { Prostate cancer } \\ \text { BCa } & \text { Bladder cancer } \\ \text { RCC } & \text { Renal cell carcinoma } \\ \text { RCa } & \text { Renal cancer } \\ \text { ROS } & \text { Reactive oxygen species } \\ \text { GSH } & \text { Glutathione } \\ \text { PSA } & \text { Prostate-specific antigen } \\ \text { BCNU } & \text { Carmustine } \\ \text { CCA } & \text { Clear cell adenocarcinoma } \\ \text { SNP } & \text { Single nucleotide polymorphism } \\ \text { SBCa } & \text { Superficial bladder cancer } \\ \text { IBCa } & \text { Invasive bladder cancer } \\ \text { TCa } & \text { Testis cancer }\end{array}$

\section{References}

1. Torre, L.A.; Bray, F.; Siegel, R.L.; Ferlay, J.; Lortet-Tieulent, J.; Jemal, A. Global cancer statistics, 2012. CA Cancer J. Clin. 2015, 65, 87-108. DOI: 10.3322/caac.21262.

2. Siegel, R.L.; Miller, K.D.; Jemal, A. Cancer statistics, 2016. CA Cancer J. Clin. 2016, 66, 7-30. DOI: 10.3322/caac.21332.

3. Liu, X.; $\mathrm{Wu}, \mathrm{Q} . ; \mathrm{Li}, \mathrm{L}$. Functional and therapeutic significance of EZH2 in urological cancers. Oncotarget 2017, 8,38044-38055. DOI: 10.18632/oncotarget.16765.

4. Ferlay, J.; Shin, H.R.; Bray, F.; Forman, D.; Mathers, C.; Parkin, D.M. Estimates of worldwide burden of cancer in 2008: GLOBOCAN 2008. Int. J. Cancer. 2010, 127, 2893-2917. DOI: 10.1002/ijc.25516.

5. Kahn, B.; Collazo, J., Kyprianou, N. Androgen receptor as a driver of therapeutic resistance in advanced prostate cancer. Int. J. Biol. Sci. 2014, 10, 588-595. DOI: 10.7150/ijbs.8671.

6. Ploeg, M.; Aben, K.K.; Kiemeney, L.A. The present and future burden of urinary bladder cancer in the world. World J. Urol. 2009, 27, 289-293. DOI: 10.1007/s00345-009-0383-3.

7. Siegel, R.; Naishadham, D., Jemal, A. Cancer statistics, 2013. CA Cancer J. Clin. 2013, 63, 11-30. DOI: 10.3322/caac.21166.

8. Sun, M., Thuret, R.; Abdollah, F., Lughezzani, G.; Schmitges, J.; Tian, Z.; Shariat, S.F.; Montorsi, F.; Patard, J.J.; Perrotte, P.; et al. Age-adjusted incidence, mortality, and survival rates of stage-specific renal cell 
9 of 12

carcinoma in North America: a trend analysis. Eur. Urol. 2011, 59, 135-141. DOI: 10.1016/j.eururo.2010.10.029.

9. Chin, A.I., Lam, J.S.; Figlin, R.A.; Belldegrun, A.S. Surveillance strategies for renal cell carcinoma patients following nephrectomy. Rev. Urol. 2006, 8, 1-7.

10. Udensi, U.K.; Tchounwou, P.B. Oxidative stress in prostate hyperplasia and carcinogenesis. J. Exp. Clin. Cancer Res. 2016, 35, 139. DOI: 10.1186/s13046-016-0418-8.

11. Gakis, G. The role of inflammation in bladder cancer. Adv. Exp. Med. Biol. 2014, 816, 183-196. DOI: 10.1007/978-3-0348-0837-8_8.

12. Yang, S.; Pinney, S.M.; Mallick, P.; Ho, S.M.; Bracken, B., Wu, T. Impact of Oxidative Stress Biomarkers and Carboxymethyllysine (an Advanced Glycation End Product) on Prostate Cancer: A Prospective Study. Clin. Genitourin. Cancer. 2015, 13, e347-351. DOI: 10.1016/j.clgc.2015.04.004.

13. Elangovan, I.; Thirugnanam, S.; Chen, A.; Zheng, G., Bosland, M.C.; Kajdacsy-Balla, A.; Gnanasekar, M. Targeting receptor for advanced glycation end products (RAGE) expression induces apoptosis and inhibits prostate tumor growth. Biochem. Biophys. Res. Commun. 2012, 417, 1133-1138. DOI: 10.1016/j.bbrc.2011.12.060.

14. Guo, Y.; Xia, P.; Zheng, J.J.; Sun, X.B.; Pan, X.D.; Zhang, X.; Wu, C.Z. Receptors for advanced glycation end products (RAGE) is associated with microvessel density and is a prognostic biomarker for clear cell renal cell carcinoma. Biomed. Pharmacother. 2015, 73, 147-153. DOI: 10.1016/j.biopha.2015.06.006.

15. Hirakawa, Y.; Inagi, R. Glycative Stress and Its Defense Machinery Glyoxalase 1 in Renal Pathogenesis. Int. J. Mol. Sci. 2017, 18, pii: E174. DOI: 10.3390/ijms18010174.

16. Mikhaylova, O.; Stratton, Y.; Hall, D.; Kellner, E.; Ehmer, B.; Drew, A.F.; Gallo, C.A.; Plas, D.R.; Biesiada, J., Meller, J.; et al. VHL-regulated MiR-204 suppresses tumor growth through inhibition of LC3B-mediated autophagy in renal clear cell carcinoma. Cancer Cell 2012, 21, 532-546. DOI: 10.1016/j.ccr.2012.02.019.

17. Solomon, D.A.; Kim, J.S.; Bondaruk, J.; Shariat, S.F.; Wang, Z.F.; Elkahloun, A.G.; Ozawa, T.; Gerard, J.; Zhuang, D.; Zhang, S.; et al. Frequent truncating mutations of STAG2 in bladder cancer. Nat. Genet. 2013, 45, 1428-1430. DOI: 10.1038/ng.2800.

18. Attard, G.; Parker, C.; Eeles, R.A.; Schröder, F.; Tomlins, S.A.K; Tannock, I., Drake, C.G.; de Bono, J.S. Prostate cancer. Lancet 2016, 387, 70-82. DOI: 10.1016/S0140-6736(14) 61947-4.

19. Rabbani, N.; Thornalley, P.J. Dicarbonyl stress in cell and tissue dysfunction contributing to ageing and disease. Biochem. Biophys. Res. Commun. 2015, 458, 221-226. DOI: 10.1016/j.bbrc.2015.01.140.

20. Ward, P.S., Thompson, C.B. Metabolic reprogramming: a cancer hallmark even warburg did not anticipate. Cancer Cell. 2012, 21, 297-308. DOI: 10.1016/j.ccr.2012.02.014.

21. Bellahcène, A.; Nokin, M.J.; Castronovo, V.; Schalkwijk, C. Methylglyoxal-derived stress: An emerging biological factor involved in the onset and progression of cancer. Semin. Cancer Biol. 2017 pii: S1044579X(17)30087-1. DOI: 10.1016/j.semcancer.2017.05.010.

22. Roy, A.; Ahir, M.; Bhattacharya, S.; Parida, P.K.; Adhikary, A.; Jana, K.; Ray, M. Induction of mitochondrial apoptotic pathway in triple negative breast carcinoma cells by methylglyoxal via generation of reactive oxygen species. Mol. Carcinog. 2017. DOI: 10.1002/mc.22665.

23. Vulesevic, B.; McNeill, B.; Giacco, F.; Maeda, K.; Blackburn, N.J.; Brownlee, M.; Milne, R.W.; Suuronen, E.J. Methylglyoxal-Induced Endothelial Cell Loss and Inflammation Contribute to the Development of Diabetic Cardiomyopathy. Diabetes. 2016, 65, 1699-1713. DOI: 10.2337/db15-0568.

24. Antognelli, C., Mezzasoma, L.; Fettucciari, K.; Talesa, V.N. A novel mechanism of methylglyoxal cytotoxicity in prostate cancer cells. Int. J. Biochem. Cell. Biol. 2013, 45, 836-844. DOI: 10.1016/j.biocel.2013.01.003.

25. Antognelli, C.; Mezzasoma, L.; Fettucciari, K.; Mearini, E.; Talesa, V.N. Role of glyoxalase I in the proliferation and apoptosis control of human LNCaP and PC3 prostate cancer cells. Prostate 2013, 73, 121132. DOI: $10.1002 /$ pros.22547.

26. Antognelli, C.; Palumbo, I.; Aristei, C.; Talesa, V.N. Glyoxalase I inhibition induces apoptosis in irradiated MCF-7 cells via a novel mechanism involving Hsp27, p53 and NF- $\kappa B$. Br J Cancer 2014, 111, 395-406. DOI: 10.1038/bjc.2014.280.

27. Antognelli, C.; Gambelunghe, A.; Muzi, G.; Talesa, V.N. Glyoxalase I drives epithelial-to-mesenchymal transition via argpyrimidine-modified Hsp70, miR-21 and SMAD signalling in human bronchial cells 
BEAS-2B chronically exposed to crystalline silica Min-U-Sil 5: Transformation into a neoplastic-like phenotype. Free Radic Biol Med. 2016, 92, 110-125. DOI: 10.1016/j.freeradbiomed.2016.01.009.

28. Rabbani, N.; Xue, M.; Weickert, M.O.; Thornalley, P.J. Multiple roles of glyoxalase 1-mediated suppression of methylglyoxal glycation in cancer biology-Involvement in tumour suppression, tumour growth, multidrug resistance and target for chemotherapy. Semin Cancer Biol. 2017, pii: S1044-579X(17)30035-4. DOI: 10.1016/j.semcancer.2017.05.006.

29. Chiavarina, B.; Nokin, M.J.; Durieux, F.; Bianchi, E.; Turtoi, A.; Peulen, O.; Peixoto, P.; Irigaray, P.; Uchida, K.; Belpomme, D.; et al. Triple negative tumors accumulate significantly less methylglyoxal specific adducts than other human breast cancer subtypes. Oncotarget 2014, 5, 5472-5482.

30. Rulli, A.; Carli, L.; Romani, R.; Baroni, T.; Giovannini, E.; Rosi, G.; Talesa, V. Expression of glyoxalase I and II in normal and breast cancer tissues. Breast Cancer Res Treat. 2001, 66, 67-72.

31. Rulli, A.; Antognelli, C.; Prezzi, E.; Baldracchini, F.; Piva, F.; Giovannini, E.; Talesa, V. A possible regulatory role of 17beta-estradiol and tamoxifen on glyoxalase I and glyoxalase II genes expression in MCF7 and BT20 human breast cancer cells. Breast Cancer Res Treat. 2006, 96, 187-196.

32. Cheng, W.; Tsai, M.M.; Tsai, C.Y.; Huang, Y.H.; Chen, C.Y.; Chi, H.C.; Tseng, Y.H.; Chao, I.W.; Lin, W.C.; Wu, S.M.; et al. Glyoxalase-I is a novel prognosis factor associated with gastric cancer progression. PLoS One 2012, 7:e34352. DOI: 10.1371/journal.pone.0034352.

33. Mearini, E.; Romani, R.; Mearini, L.; Antognelli, C.; Zucchi, A.; Baroni, T.; Porena, M.; Talesa, V.N. Differing expression of enzymes of the glyoxalase system in superficial and invasive bladder carcinomas. Eur J Cancer 2002, 38, 1946-1950.

34. Antognelli, C.; Baldracchini, F.; Talesa, V.N.; Costantini, E.; Zucchi, A.; Mearini, E. Overexpression of glyoxalase system enzymes in human kidney tumor. Cancer J. 2006, 12, 222-228.

35. Antognelli, C.; Del Buono, C.; Baldracchini, F.; Talesa, V.; Cottini, E.; Brancadoro, C.; Zucchi, A.; Mearini, E. Alteration of glyoxalase genes expression in response to testosterone in $\mathrm{LNCaP}$ and $\mathrm{PC} 3$ human prostate cancer cells. Cancer Biol Ther. 2007, 6, 1880-1888.

36. Talesa, V.N.; Ferri, I.; Bellezza, G.; Love, H.D.; Sidoni, A.; Antognelli C. Glyoxalase 2 Is Involved in Human rostate Cancer Progression as Part of a Mechanism Driven By PTEN/PI3K/AKT/mTOR Signaling With Involvement of PKM2 and ER $\alpha$. Prostate 2017, 77, 196-210. DOI: 10.1002/pros.23261.

37. Antognelli C, Ferri I, Bellezza G, Siccu P, Love HD, Talesa VN, Sidoni A. Glyoxalase 2 drives tumorigenesis in human prostate cells in a mechanism involving androgen receptor and p53-p21 axis. Mol Carcinog. 2017, 56, 2112-2126. doi: 10.1002/mc.22668. DOI: 10.1002/mc.22668.

38. de Hemptinne, V.; Rondas, D.; Toepoel, M.; Vancompernolle, K. Phosphorylation on Thr-106 and NOmodification of glyoxalase I suppress the TNF-induced transcriptional activity of NF-kappaB. Mol Cell Biochem. 2009, 325, 169-178. DOI: 10.1007/s11010-009-0031-7.

39. Van Herreweghe, F.; Mao, J., Chaplen, F.W.; Grooten, J.; Gevaert, K.; Vandekerckhove, J.; Vancompernolle, K. Tumor necrosis factor-induced modulation of glyoxalase I activities through phosphorylation by PKA results in cell death and is accompanied by the formation of a specific methylglyoxal-derived AGE. Proc Natl Acad Sci USA. 2002, 99, 949-954.

40. de Hemptinne, V.; Rondas, D.; Vandekerckhove, J.; Vancompernolle, K. Tumour necrosis factor induces phosphorylation primarily of the nitric-oxide-responsive form of glyoxalase I. Biochem J. 2007, 407, 121-128.

41. Birkenmeier, G., Stegemann, C.; Hoffmann, R.; Günther, R.; Huse, K.; Birkemeyer C. Posttranslational modification of human glyoxalase 1 indicates redox-dependent regulation. PLoS One 2010, 5:e10399. DOI: 10.1371/journal.pone.0010399.

42. Xu, Y.; Chen, X. Glyoxalase II, a detoxifying enzyme of glycolysis byproduct methylglyoxal and a target of p63 and p73, is a pro-survival factor of the p53 family. J Biol Chem. 2006, 281, 26702-26713.

43. Geng, X.; Ma, J.; Zhang, F.; Xu, C. Glyoxalase I in tumor cell proliferation and survival and as a potential target for anticancer therapy. Oncol Res Treat. 2014, 37, 570-574. DOI: 10.1159/000367800.

44. Sousa Silva, M.; Gomes, R.A.; Ferreira, A.E., Ponces Freire, A.; Cordeiro, C. The glyoxalase pathway: the first hundred years... and beyond. Biochem J. 2013, 453, 1-15. DOI: 10.1042/BJ20121743.

45. Rabbani, N.; Thornalley, P.J. Glyoxalase in diabetes, obesity and related disorders. Semin Cell Dev Biol. 2011, 22, 309-317. DOI: 10.1016/j.semcdb.2011.02.015.

46. Xue, M., Rabbani, N.; Thornalley, P.J. Glyoxalase in ageing. Semin Cell Dev Biol. 2011, 22, 293-301. DOI: 10.1016/j.semcdb.2011.02.013. 
47. Rabbani, N., Thornalley, P.J. The glyoxalase system--from microbial metabolism, through ageing to human disease and multidrug resistance. Semin Cell Dev Biol. 2011, 22, 261. DOI: 10.1016/j.semcdb.2011.02.014.

48. Thornalley, P.J.; Rabbani, N. Glyoxalase in tumourigenesis and multidrug resistance. Semin Cell Dev Biol. 2011, 22, 318-325. DOI: 10.1016/j.semcdb.2011.02.006.

49. Ayoub, F.; Zaman, M., Thornalley, P.; Masters, J. Glyoxalase activities in human tumour cell lines in vitro. Anticancer Res. 1993, 13, 151-155.

50. Di Ilio, C.; Angelucci, S.; Pennelli, A.; Zezza, A.; Tenaglia, R.; Sacchetta, P. Glyoxalase activities in tumor and non-tumor human urogenital tissues. Cancer Lett. 1995, 96, 189-193.

51. Davidson, S.D.; Cherry, J.P.; Choudhury, M.S.; Tazaki, H.; Mallouh, C.; Konno, S. Glyoxalase I activity in human prostate cancer: a potential marker and importance in chemotherapy. J Urol. 1999, 161, 690-691.

52. Davidson, S.D.; Milanesa, D.M.; Mallouh, C.; Choudhury, M.S.; Tazaki, H.; Konno, S. A possible regulatory role of glyoxalase I in cell viability of human prostate cancer. Urol Res. 2002, 30, 116-121.

53. Baunacke, M.; Horn, L.C.; Trettner, S., Engel, K.M.; Hemdan, N.Y.; Wiechmann, V.; Stolzenburg, J.U.; Bigl, M.; Birkenmeier, G. Exploring glyoxalase 1 expression in prostate cancer tissues: targeting the enzyme by ethyl pyruvate defangs some malignancy-associated properties. Prostate 2014, 74, 48-60. DOI: 10.1002/pros.22728.

54. Sakamoto, H.; Mashima, T.; Sato, S.; Hashimoto, Y.; Yamori, T.; Tsuruo, T. Selective activation of apoptosis program by S-p-bromobenzylglutathione cyclopentyl diester in glyoxalase I-overexpressing human lung cancer cells. Clin Cancer Res. 2001, 7, 2513-2518.

55. Samadi, A.A.; Fullerton, S.A.; Tortorelis, D.G.; Johnson, G.B.; Davidson, S.D.; Choudhury, M.S.; Mallouh, C.; Tazaki, H.; Konno, S. Glyoxalase I phenotype as a potential risk factor for prostate carcinoma. Urology 2001, 57, 183-187.

56. Meehan, K.L.; Holland, J.W.; Dawkins, H.J. Proteomic analysis of normal and malignant prostate tissue to identify novel proteins lost in cancer. Prostate 2002, 50, 54-63.

57. Chavan, S.V.; Chavan, N.R.; Balaji, A.; Trivedi, V.D.; Chavan, P.R. A pilot study on the use of serum glyoxalase as a supplemental biomarker to predict malignant cases of the prostate in the PSA range of 4-20 ng/ml. Indian J Med Res. 2011, 134, 458-462.

58. Antognelli, C.; Mezzasoma, L.; Mearini, E.; Talesa, V.N. Glyoxalase 1-419C>A variant is associated with oxidative stress: implications in prostate cancer progression. PLoS One 2013, 8:e74014. DOI: 10.1371/journal.pone.0074014.

59. Catalona, W.J.; Southwick, P.C.; Slawin, K.M.; Partin, A.W., Brawer, M.K.; Flanigan, R.C.; Patel, A.; Richie, J.P.; Walsh, P.C.; Scardino, P.T.; et al. Comparison of percent free PSA, PSA density, and age-specific PSA cutoffs for prostate cancer detection and staging. Urology 2000, 56, 255-260.

60. Talesa, V.N.; Antognelli, C.; Del Buono, C., Stracci, F.; Serva, M.R.; Cottini, E.; Mearini E. Diagnostic potential in prostate cancer of a panel of urinary molecular tumor markers. Cancer Biomark. 2009, 5, 241251. DOI: 10.3233/CBM-2009-0109.

61. Mearini, E.; Antognelli, C.; Del Buono, C., Cochetti, G.; Giannantoni, A.; Nardelli, E.; Talesa, V.N. The combination of urine DD3(PCA3) mRNA and PSA mRNA as molecular markers of prostate cancer. Biomarkers 2009, 14, 235-243. DOI: 10.1080/13547500902807306.

62. Sharkey, E.M.; O'Neill, H.B.; Kavarana, M.J.; Wang, H.; Creighton, D.J.; Sentz, D.L.; Eiseman, J.L. Pharmacokinetics and antitumor properties in tumor-bearing mice of an enediol analogue inhibitor of glyoxalase I. Cancer Chemother Pharmacol. 2000; 46, 156-166.

63. Finkelstein, M.P.; Aynehchi, S.; Samadi, A.A.; Drinis, S.; Choudhury, M.S.; Tazaki, H.; Konno, S.J. Chemosensitization of carmustine with maitake beta-glucan on androgen-independent prostatic cancer cells: involvement of glyoxalase I. Altern Complement Med. 2002, 8, 573-580.

64. Santel, T., Pflug, G.; Hemdan, N.Y.; Schäfer, A.; Hollenbach, M.; Buchold, M.; Hintersdorf, A., Lindner, I.; Otto, A.; Bigl, M.; Oerlecke, I.; et al. Curcumin inhibits glyoxalase 1: a possible link to its antiinflammatory and anti-tumor activity. PLoS One 2008, 3:e3508. DOI: 10.1371/journal.pone.0003508.

65. Valenti, D.; Vacca, R.A.; de Bari, L. 3-Bromopyruvate induces rapid human prostate cancer cell death by affecting cell energy metabolism, GSH pool and the glyoxalase system. J Bioenerg Biomembr. 2015, 47, 493506. DOI: 10.1007/s10863-015-9631-y.

66. Rubin, M.A., Maher, C.A.; Chinnaiyan, A.M. Common gene rearrangements in prostate cancer. J Clin Oncol 2011, 29, 3659-3668. DOI: 10.1200/JCO.2011.35.1916. 
67. Fabris, L.; Ceder, Y.; Chinnaiyan, A.M.; Jenster, G.W.; Sorensen, K.D.; Tomlins, S., Visakorpi, T.; Calin, G.A. The potential of MicroRNAs as prostate cancer biomarkers. Eur Urol 2016, pii:S0302-2838(16)00012-9.

68. Sailer V, Stephan C, Wernert N, Perner S, Jung K, Dietel M, Kristiansen, G. Comparison of p40 $(\Delta \mathrm{Np63})$ and p63 expression in prostate tissues-which one is the superior diagnostic marker for basal cells? Histopathology 2013, 63, 50-56. DOI: 10.1111/his.12116.

69. Tanaka, T.; Kuramitsu, Y.; Wang, Y.; Baron, B.; Kitagawa, T.; Tokuda, K.; Hirakawa, K.; Yashiro, M.; Naito, S.; Nakamura, K. Glyoxalase 1 as a candidate for indicating the metastatic potential of of SN12Chuman renal cell carcinoma cell clones. Oncol Rep. 2013, 30, 2365-2370. DOI: 10.3892/or.2013.2699.

70. Rabbani, N.; Xue, M.; Thornalley, P.J. Methylglyoxal-induced dicarbonyl stress in aging and disease: first steps towards glyoxalase 1-based treatments. Clin Sci (Lond). 2016, 130, 1677-1696. DOI: 10.1042/CS20160025.

71. Chocholatý, M.; Jáchymová, M.; Schmidt, M.; Havlová, K.; Křepelová, A.; Zima, T.; Babjuk, M.; Kalousová, M. Polymorphisms of the receptor for advanced glycation end-products and glyoxalase I in patients with renal cancer. Tumour Biol. 2015, 36, 2121-2126. DOI: 10.1007/s13277-014-2821-0.

72. Truta, A.; Popon, T.A.H.; Saraci, G.; Ghervan, L.; Pop, I.V. Health Related Quality of life in bladder cancer. Current approach and future perspectives. Clujul Med. 2017, 90, 262-267. DOI: 10.15386/cjmed-693.

73. Lin, S.Y.; Linehan, J.A.; Wilson, T.G.; Hoon, D.S.B. Emerging Utility of Urinary Cell-free Nucleic Acid Biomarkers for Prostate, Bladder, and Renal Cancers. Eur Urol Focus. 2017, pii: S2405-4569(17)300779. DOI: 10.1016/j.euf.2017.03.009.

74. Costa, A.L.; Lobo, J.; Jerónimo, C.; Henrique, R. The epigenetics of testicular germ cell tumors: looking for novel disease biomarkers. Epigenomics 2017, 9, 155-169. DOI: 10.2217/epi-2016-0081.

75. Chieffi, P. An Overview on Predictive Biomarkers of Testicular Germ Cell Tumors. J Cell Physiol 2017, 232, 276-280. DOI: $10.1002 /$ jcp.25482. 